\title{
Correlative Imaging and Spectroscopy of Particles in Liquid
}

Xiao-Ying $\mathrm{Yu}^{1}$, Bruce Arey ${ }^{2}$, Hee Joon Jung ${ }^{2}$, Libor Kovarik ${ }^{3}$, Zihua Zhu ${ }^{3}$, Juan Yao ${ }^{1}$, Jiachao Yu ${ }^{1}$, Xiao Sui ${ }^{1}$, Tyler Troy ${ }^{4}$, Biswajit Bandyopadhyay ${ }^{4}$, and Musa Ahmed ${ }^{4}$

1. Earth and Biological Sciences Directorate, Pacific Northwest National Laboratory (PNNL), Richland, WA 99354, USA.

2. Energy and Environment Directorate, PNNL, WA 99354, USA.

3. W. R \& Wiley Environmental Molecular Science Laboratory, PNNL, Richland, WA 99354, USA.

4. Chemical Sciences Division, Lawrence Berkeley National Laboratory, Berkeley, CA 94720, USA.

Correlative imaging and spectroscopy has been widely used in biological and medical sciences. Its power of providing a holistic view of the system of interest makes it an intense research topic that often utilizes correlative optical microscopy, cryo electron microscopy, and a variety of spectroscopy techniques. With the advent of liquid handling in vacuum, in situ electron microscopy has become increasingly popular in studying complex systems, adding another palette in correlative imaging and spectroscopy. This work presents an example of characterization of particles suspended in liquid in a vacuum compatible microfluidic sample holder using a suite of tools including scanning electron microscopy (SEM), transmission electron microscopy (TEM), time-of-flight secondary ion mass spectrometry (ToF-SIMS), and synchrotron vacuum UV (VUV) single photon ionization mass spectrometry (SPI-MS), highlighting the advantage of multiscale analysis in material sciences.

We developed a transferrable and vacuum compatible microfluidic interface, System for Analysis at the Liquid Vacuum Interface (SALVI), which enabled surface analysis of liquids and liquid-solid interactions using ToF-SIMS and SEM [1, 2]. Its detection window is an aperture of 2-3 $\mu \mathrm{m}$ in diameter open to vacuum, which allows direct detection of the liquid surface. Liquid is withheld by surface tension within the aperture. The interface is composed of a silicon nitride (SiN) membrane and polydimethylsiloxane (PDMS) microchannel [1, 2]. It has been used in a variety of applications including complex liquid mixtures, ionic liquids, single mammalian cells, live biofilms, and solidelectrolyte interface (SEI) [3,4]. This paper shows the first results of multimodal analysis of relatively large polydisperse particles (i.e., boehmite with $99.9 \%$ AlOOH and $\mathrm{D}_{50}$ of $800 \mathrm{~nm}$, Nabaltec, APYRAL AOH60) suspended in deionized (DI) water.

Both high vacuum and low vacuum conditions were employed in liquid SEM (FEI Quanta). Figure 1 depicts SEM imaging results of boehmite particles in the microchannel. Both secondary electron (SE) and backscattered electron (BSE) mode can be used to image particle size and morphology. While electron microscopy provides a fine description of particle size, shape, morphology, and crystal structure at the single particle level enhanced with elemental mapping, particle molecular structure and composition are missing. Neither is the solvent microenvironment observable using electron microscopy. Therefore, imaging mass spectrometry and synchrotron-based energy dependent mass spectrometry are used to fill in the knowledge gap. Figure 2 depicts a comparison of positive ion mass spectra acquired using in situ liquid ToF-SIMS (IONTOF GmbH, TOF-SIMS V) and liquid VUV SPIMS (ALS 9.0.2). There is a difference in the $\mathrm{m} / \mathrm{z}$ spectra due to the ionization mechanism distinction between SIMS and SPI-MS. SIMS provides detailed submicron molecular mapping of the particle and its surrounding water cluster environments as well as molecular identification of small molecules in liquid. SPI-MS has been considered as the long-sought universal soft ionization (or fragment free) method, offering the capability to detect all organic molecules [5]. It provides quantitative analysis of 
components based on ionization energy (IE) and appearance onsets of fragments according to the photoionization efficiency (PIE) spectrum. These new results demonstrate advancements of in situ correlative imaging of liquid surfaces and solid-liquid interfaces using a universal microfluidic interface, SALVI [6].

\section{References:}

[1] L Yang et al., J. Vac. Sci. Technol. A 29 (2011), art. no., 061101. doi: 10.1116/1.3654147.

[2] L Yang et al., Lab Chip 11 (2011), 2481-4. doi: 10.1039/c01c00676a.

[3] H Xin et al., Analyst (2014), 139(7), 1609-13. doi: 10.1039/C3AN02262E.

[4] B Liu et al., Lab Chip. (2014), 14(5), 854-859. doi: 10.1039/C3LC50971K.

[5] L. Hanley and R. Zimmerman, Anal. Chem. (2009), 81, 4174-82. doi: 10.1021/ac8013675.

[6] H Shi et al., Catal. Sci. Technol. (2015), 5, 3035-60. doi: 10.1039/C4CY01720J.

[7] We acknowledge support from the PNNL Technology Investment Council (TIC) Program, Materials Synthesis and Simulation across Scales $\left(\mathrm{MS}^{3}\right)$ Initiative, and the Nuclear Science Processing Initiative (NPSI) Laboratory Directed Research and Development fund and instrument access to the DOE BER EMSL and BES ALS user facilities.
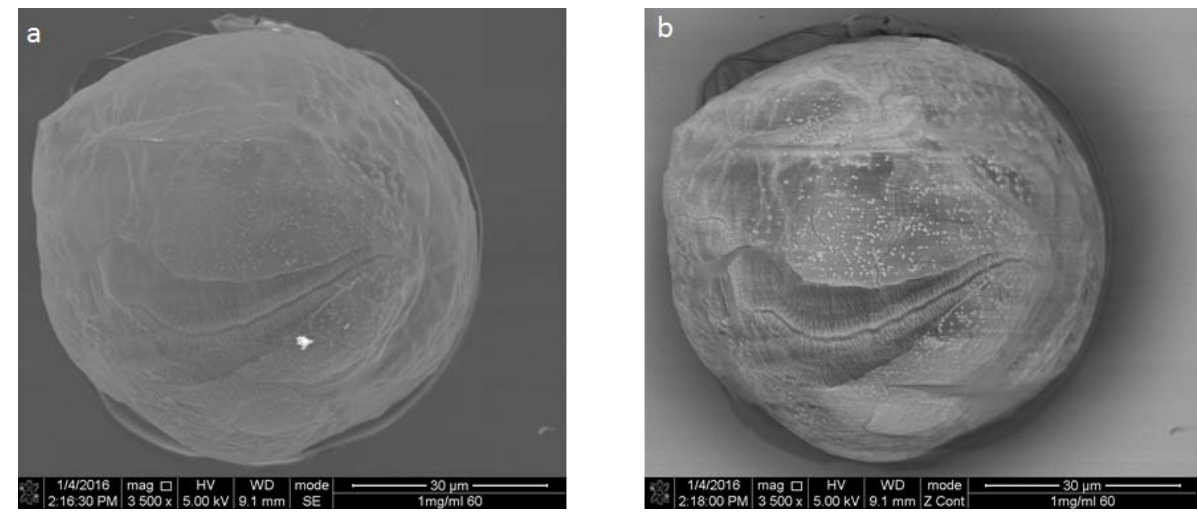

Figure 1. Liquid SEM imaging of boehmite particles in DI water: (a) secondary electrons and (b) backscattered electrons acquired under low vacuum (0.1 - 0.16 mbar) conditions in the FEI Quanta.
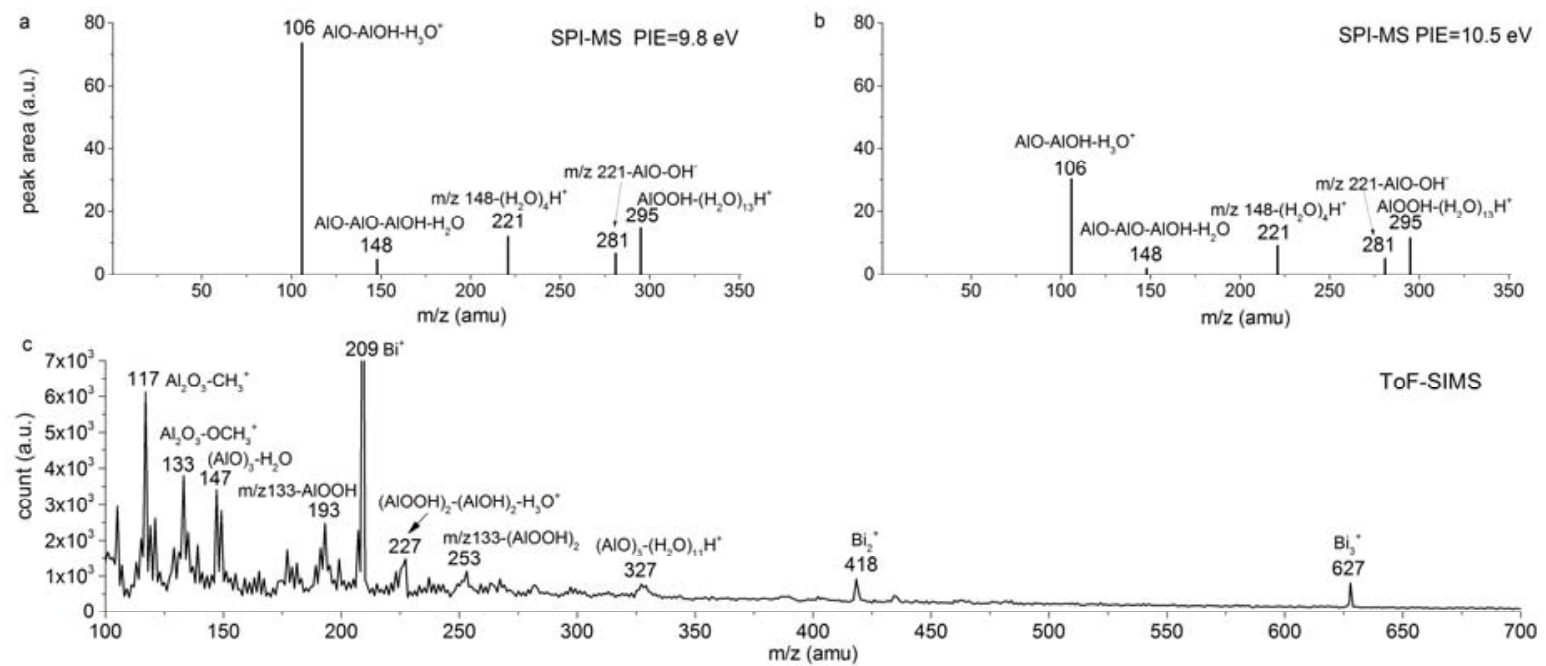

Figure 2. Positive $\mathrm{m} / \mathrm{z}$ spectrum of boehmite particles in DI water acquired by (a) SPI-MS at PIE=9.8 $\mathrm{eV}$, (b) SPI-MS at PIE=10.5 eV, and (c) ToF-SIMS. 